\title{
Towards an all-integrated MOPA configuration using Yb- doped ion-exchanged waveguides
}

\author{
Amol Choudhary, ${ }^{1 *}$ Alexander A.Lagatsky, ${ }^{2}$ Pradeesh Kannan ${ }^{1}$, Christian T.A.Brown ${ }^{3}$ and David P.Shepherd ${ }^{1}$ \\ ${ }^{1}$ Optoelectronics Research Centre, University of Southampton, Southampton, SO17 1BJ, United Kingdom \\ ${ }^{2}$ Fraunhofer Centre for Applied Photonics, Glasgow G1 2TB, United Kingdom \\ ${ }^{3}$ School of Physics and Astronomy, University of St Andrews, St Andrews, KY16 9SS, United Kingdom \\ *Authore-mail address: a.choudhary@soton.ac.uk
}

\begin{abstract}
In this paper, we present an ion-exchanged Yb-glass waveguide amplifier, seeded by an ion-exchanged $\mathrm{Yb}$-glass waveguide laser demonstrating a gain as high as $10 \mathrm{~dB}$. We also present multi-GHz, mode-locked ion-exchanged waveguide lasers and discuss the development of a fully integrated high-power, multi-GHz waveguide source.

OCIS codes: (230.7380) Waveguides, channeled; Laser amplifiers; (140.4050); Mode-locked lasers; (140.3480) Lasers, diode-pumped; (140.3280); (140.3615) Lasers, ytterbium
\end{abstract}

\section{Introduction}

Femtosecond lasers with high repetition rates in excess of $1 \mathrm{GHz}$ can have key applications in optical frequency comb metrology and in the calibration of astronomical spectrographs where the high power per comb line can result in increased resolution, in non-linear optical microscopy where the high number of optical pulses per second (high brightness at reduced pulse energy) could be beneficial for a high signal to noise ratio, and for optical sampling. Typically, the laser cavity length needs to be reduced below $15 \mathrm{~cm}$ to access the gigahertz-repetition-rate-regime, which can be quite challenging to engineer. Diode-pumped solid-state lasers in a waveguide geometry are a viable solution for developing multi-GHz sources. Waveguide lasers can have the saturable absorber and the cavity mirror integrated into one chip, which makes the cavity very compact and can be mass-produced using photonic microfabrication techniques. Owing to the small laser mode-sizes (sub-10- $\mu \mathrm{m}$ ) in both directions in a channel waveguide laser, the laser threshold can be substantially reduced and high slope efficiencies may be obtained by optimizing the waveguide fabrication technique to keep the propagation losses low. The confined laser mode-size is also tightly focused on the saturable absorber, which can reduce the mode-locking threshold. One of the first integrated modelocked waveguide lasers was limited in average power to $\sim 1 \mathrm{~mW}$ and had a repetition-rate of $400 \mathrm{MHz}$ [1]. A high power, multi-GHz waveguide laser is essential for the development of a self-referenced frequency comb.

In this paper, we discuss our work on the development of mode-locked, multi-GHz waveguide lasers and Qswitched waveguide lasers fabricated by ion-exchanging $\mathrm{Yb}$-doped phosphate glass. We also present a $2.5-\mathrm{cm}$-long ion-exchanged $\mathrm{Yb}$-doped phosphate glass waveguide amplifier, seeded by another $\mathrm{CW}$ ion-exchanged $\mathrm{Yb}$-doped phosphate glass waveguide laser in a master oscillator power amplifier configuration (MOPA), which demonstrates a gain of $4.8 \mathrm{~dB}$ at $1054 \mathrm{~nm}, 5.3 \mathrm{~dB}$ at $1046 \mathrm{~nm}$ and $10 \mathrm{~dB}$ at $1017 \mathrm{~nm}$. These results are very promising for the development of an on-chip, high-power, multi-GHz source.

\section{Experimental details}

The waveguides were fabricated in commercially available Yb:IOG-1 glass samples using ion-exchange as described in [2]. The ion-exchange was carried out in a molten salt mixture of $45 \mathrm{~mol} \% \mathrm{KNO}_{3}-50 \mathrm{~mol} \% \mathrm{NaNO}_{3}-$ $5 \mathrm{~mol} \% \mathrm{AgNO}_{3}$ at a temperature of $325^{\circ} \mathrm{C}$ for 10 minutes. The $\mathrm{Na}^{+}$ions in the glass matrix were replaced with the $\mathrm{K}^{+}$and the $\mathrm{Ag}^{+}$ions in the ion-exchange melt, resulting in the local increase of the refractive index, thus allowing waveguiding. The glass samples were polished to lengths of $25 \mathrm{~mm}, 20 \mathrm{~mm}, 17.8 \mathrm{~mm}, 9.4 \mathrm{~mm}, 8 \mathrm{~mm}$ and $6.5 \mathrm{~mm}$.

A schematic of the amplifier setup is shown in figure 1 (a). The pump and the seed were counter-propagated in the waveguide amplifier. The seed is a 17.8-mm-long Yb:IOG-1 waveguide laser which is pumped by a singlemode, fibre-coupled laser diode operating at $975 \mathrm{~nm}$. The fibre-coupled output was collimated by an aspheric lens with $\mathrm{f}=8 \mathrm{~mm}$ and launched into the waveguide using an $\mathrm{f}=11 \mathrm{~mm}$ aspheric lens. A half-wave plate and a Faraday isolator were also installed in the pump beam path to protect the laser diode from back-reflections. A thin $(<200 \mu \mathrm{m})$ dielectric mirror with $\mathrm{T}=99 \%$ at $975 \mathrm{~nm}$ and $\mathrm{T}<0.1 \%$ between $1015-1060 \mathrm{~nm}$ was butted on to the input facet, and different output coupling mirrors were end-butted at the output facet to select different wavelengths. The output from the waveguide laser was collected by an $\mathrm{f}=11 \mathrm{~mm}$ aspheric lens and the laser and pump beams were separated by a dichroic mirror. An optical isolator was installed to prevent laser feedback into the waveguide laser and a variable attenuator was used to control the seed power launched into the amplifier. The beam was launched into the 25-mm-long Yb-doped glass waveguide amplifier using an $\mathrm{f}=11 \mathrm{~mm}$ aspheric lens and the output was also 
collimated by an $\mathrm{f}=11 \mathrm{~mm}$ aspheric lens. A fibre-coupled pump laser diode was collimated by an $8 \mathrm{~mm}$ aspheric lens which was launched into the amplifier waveguide using an $\mathrm{f}=11 \mathrm{~mm}$ aspheric lens to set up a counter-propagating cavity as shown in figure 1 (a). A dichroic mirror was installed between the two lenses to separate the pump and the seed beams. The beam profile of the transmitted seed through the waveguide amplifier with and without the pump is shown in figure 1. (b). It can be seen that no substantial change in the $1 / \mathrm{e}^{2}$ mode sizes are observed by switching the pump on.

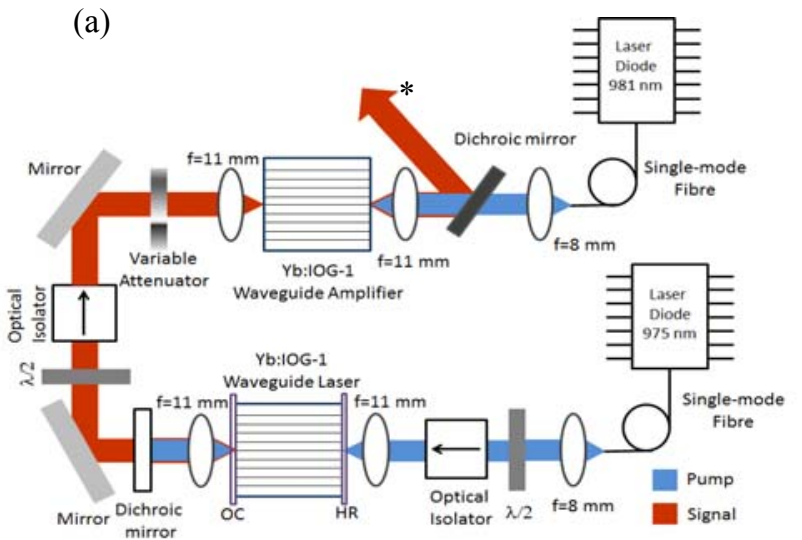

(b) No Pump

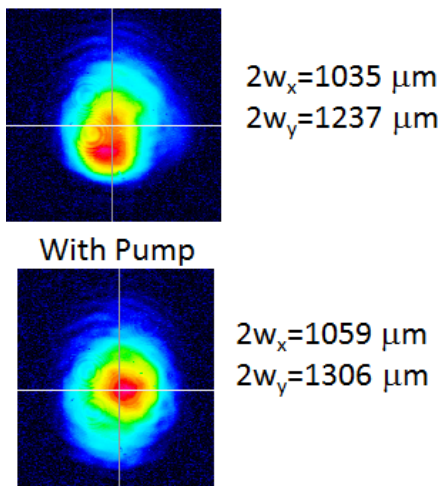

Fig. 1. (a) Experimental setup for the amplification of the waveguide laser seed (b) measured signal profiles with and without the pump, measured at the location marked with * in Fig. 1. (a).

The $20 \mathrm{~mm}, 9.4 \mathrm{~mm}, 8 \mathrm{~mm}$ and $6.5 \mathrm{~mm}$ long samples were mode-locked using SESAMs and the $17.8 \mathrm{~mm}$ long sample was Q-switched using graphene. The results have been summarised in Table 1.

Table 1. Summary of pulsed Ion-exchanged Yb-glass waveguide lasers.

\begin{tabular}{|c|c|c|c|c|c|c|}
\hline $\begin{array}{c}\text { Sample } \\
\text { length } \\
\mathbf{( m m}\end{array}$ & $\begin{array}{c}\text { Saturable } \\
\text { absorber }\end{array}$ & $\begin{array}{c}\text { Repetition- } \\
\text { rate }\end{array}$ & $\begin{array}{c}\text { Pulse } \\
\text { Duration } \\
\text { wavelength } \\
\mathbf{( n m}\end{array}$ & $\begin{array}{c}\text { Centre } \\
\text { bandwidth } \\
\text { product }\end{array}$ & $\begin{array}{c}\text { Time- } \\
\text { power } \\
(\mathbf{m W})\end{array}$ \\
\hline 20 & SESAM & $4.9 \mathrm{GHz}$ & $800 \mathrm{fs}$ & 1052 & 0.46 & $81[2]$ \\
\hline 9.4 & SESAM & $10.4 \mathrm{GHz}$ & $757 \mathrm{fs}$ & 1041.4 & 0.56 & $60[3]$ \\
\hline 8 & SESAM & $12 \mathrm{GHz}$ & $824 \mathrm{fs}$ & 1045.7 & 0.43 & $45[3]$ \\
\hline 6.5 & SESAM & $15.2 \mathrm{GHz}$ & $811 \mathrm{fs}$ & 1047.4 & 0.49 & $27[3]$ \\
\hline 17.8 & Graphene & $833 \mathrm{kHz}$ & $140 \mathrm{~ns}$ & 1057.0 & Q-switched & $21[4]$ \\
\hline
\end{tabular}

\section{Results}

A 5\% output coupling mirror (OC) was used in the seed and lasing occurred at $1054 \mathrm{~nm}$ which corresponds to the lasing wavelength of the $4.9 \mathrm{GHz}$ oscillator and the Q-switched oscillator and a $10 \% \mathrm{OC}$ was used to shift the wavelength to $1046 \mathrm{~nm}$ which corresponds to the higher repetition-rates as seen from table 1 . The Fresnel reflections (OC $96 \%$ ) from the waveguide facet were used to provide feedback to the laser cavity which allowed laser operation at $1017 \mathrm{~nm}$. This wavelength could be of interest for core-pumping of amplifier systems [5]. The seed output was launched into the amplifier and the results of the output power as a function of launched pump power for different seed powers is shown in figure 2. It can be seen on increasing the pump power the output power increases, however, there are saturation effects observed at higher pump powers.

The output powers vs. launched signal powers at maximum pump powers are plotted in figure 3 (a) for all three wavelengths. At $1017 \mathrm{~nm}$, strong saturation effects are observed and a maximum output power of $234 \mathrm{~mW}$ is obtained at an incident power of $45 \mathrm{~mW}$. The $1046 \mathrm{~nm}$ and $1054 \mathrm{~nm}$ operate in the linear regime with an output power of $72 \mathrm{~mW}$ being obtained for an input power of $27 \mathrm{~mW}$ at $1046 \mathrm{~nm}$ and an output power of $84 \mathrm{~mW}$ being obtained for an input power of $33 \mathrm{~mW}$ at $1054 \mathrm{~nm}$. The small-signal, single-pass gain as a function of launched 
pump power for all the three wavelengths is shown in figure 3(b). It can be seen that a maximum gain of $10 \mathrm{~dB}$ is obtained at a seed wavelength of $1017 \mathrm{~nm}$ at maximum pump power. A maximum gain of $5.3 \mathrm{~dB}$ and $4.8 \mathrm{~dB}$ is obtained at $1046 \mathrm{~nm}$ and $1054 \mathrm{~nm}$, respectively. Strong saturation can be observed in all three cases. The high loss for zero pump power in the case of $1017 \mathrm{~nm}$ can be attributed to the strong re-absorption of this wavelength in Ybphosphate glass. It can be seen that the experimental values are in good agreement with the simulated values.

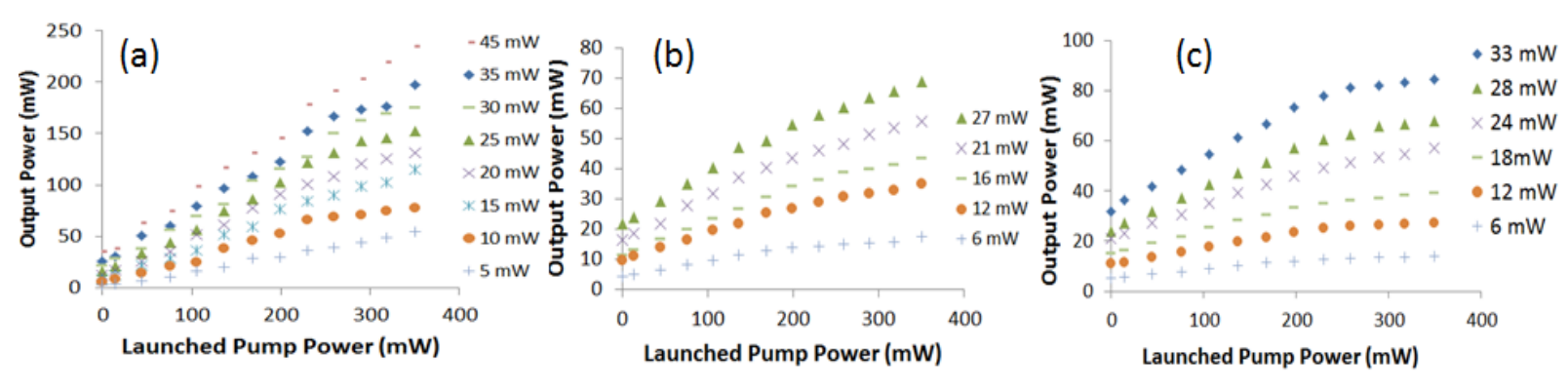

Fig. 2. Output power vs. launched pump power for different input signal powers at a signal wavelength of (a) $1017 \mathrm{~nm}$, (b)1046 nm and, (c) 1054 $\mathrm{nm}$
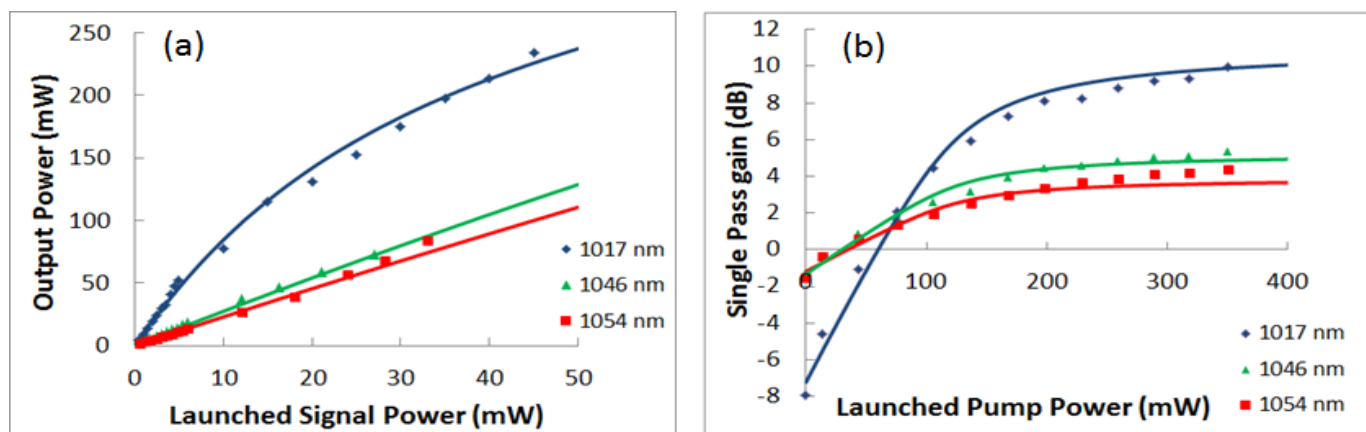

Fig. 3. (a) Output signal power vs. launched signal power for a launched pump power of $350 \mathrm{~mW}$, and (b) single-pass gain vs. launched pump power for a signal power of $0.5 \mathrm{~mW}$. Markers-experimental data, solid line-simulated values.

\section{Conclusions}

We have developed multi-GHz, mode-locked and Q-switched ion-exchanged waveguide oscillators delivering average output powers of up to $80 \mathrm{~mW}$. We also present an ion-exchanged $\mathrm{Yb}$-glass waveguide amplifier, which is seeded by an ion-exchanged Yb-glass waveguide laser. Operation at different wavelengths is studied by changing the output coupling mirror of the seed laser and a gain of as high as $10 \mathrm{~dB}$ is observed in the 2.5-long-amplifier at a wavelength of $1017 \mathrm{~nm}$. Gains of $4.8 \mathrm{~dB}$ and $5.3 \mathrm{~dB}$ are observed at $1054 \mathrm{~nm}$ and $1046 \mathrm{~nm}$, respectively. These are very promising results and amplification experiments of mode-locked and Q-switched pulses from our waveguide lasers are under progress and will be presented at the conference. It can be concluded that the construction of a mode-locked source and the amplifier on one chip is feasible, which will allow the generation of high-power, multi$\mathrm{GHz}$ pulses from an integrated device.

\section{References}

[1] H. Byun, A. Hanjani, S. Frolov, E.P. Ippen, D. Pudo, J. Shmulovich, F.X. Kartner, IEEE Photon. Tech. Lett. 21, 763-765 (2009).

[2]A. Choudhary, A.A. Lagatsky, K. Pradeesh, W. Sibbett, C.T.A. Brown and D.P. Shepherd, Opt. Lett. 37, 4416-4418 (2012).

[3]A.A. Lagatsky, A. Choudhary, P. Kannan, D.P. Shepherd, W. Sibbett, and C.T.A. Brown, Opt. Express 21, 19608-19614 (2013).

[4] A. Choudhary, S. Dhingra, B. D’Urso, K. Pradeesh and D. P. Shepherd, EPS-QEOD Europhoton Conference TuP-T2-P-05, Neuchatel, Switzerland (2014).

[5] C. Ottenhues, T. Theeg, K. Hausmann, M. Wysmolek, H. Sayinc, J. Neumann, D. Kracht, EPS-QEOD Europhoton Conference ThP-T1-P-10, Neuchatel, Switzerland (2014). 